\title{
The RHIC polarized source upgrade
}

\author{
A. Zelenski, G. Atoian, D. Steski \\ Brookhaven National Laboratory
}

V. Davydenko, A. Ivanov, A. Kolmogorov

Budker Institute of Physics

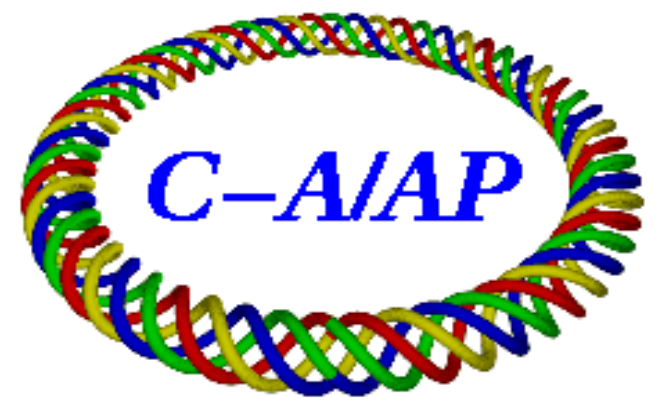

\section{Collider-Accelerator Department Brookhaven National Laboratory Upton, NY 11973}

Notice: This document has been authorized by employees of Brookhaven Science Associates, LLC under Contract No. DE-AC02-98CH10886 with the U.S. Department of Energy. The United States Government retains a nonexclusive, paid-up, irrevocable, world-wide license to publish or reproduce the published form of this document, or allow others to do so, for United States Government purposes. 


\title{
THE RHIC POLARIZED SOURCE UPGRADE
}

\author{
A.ZELENSKI, G. ATOIAN, J. RITTER, D. STESKI. \\ Brookhaven National Laboratory, Upton, NY \\ V. DAVYDENKO, A. IVANOV, A. KOLMOGOROV \\ Budker Institute for Nuclear Physics, Novosibirsk
}

\begin{abstract}
The basic limitations on the high-intensity $\mathrm{H}^{-}$ion beam production were experimentally studied in charge-exchange collisions of the neutral atomic hydrogen beam in the Na- vapor jet ionizer cell. These studies are the part of the polarized source upgrade (to $10 \mathrm{~mA}$ peak current and $85 \%$ polarization) project for RHIC. In the source the atomic hydrogen beam of a $5-10 \mathrm{keV}$ energy and total (equivalent) current up to $5 \mathrm{~A}$ is produced by neutralization of proton beam in pulsed hydrogen gas target. Formation of the proton beam (from the surface of the plasma emitter with a low transverse ion temperature $\sim 0.2 \mathrm{eV}$ ) is produced by four-electrode spherical multi-aperture ion-optical system with geometrical focusing. The hydrogen atomic beam intensity up to $1.0 \mathrm{~A} / \mathrm{cm}^{2}$ (equivalent) was obtained in the Na-jet ionizer aperture of a $2.0 \mathrm{~cm}$ diameter. At the first stage of the experiment $\mathrm{H}^{-}$beam with $36 \mathrm{~mA}$ current, $5 \mathrm{keV}$ energy and $\sim 1.0 \mathrm{~cm} \cdot \mathrm{mrad}$ normalized emittance was obtained using the flat grids and magnetic focusing. The experimental results of the high-intensity neutral hydrogen beam generation and studies of the charge-exchange polarization processes of this intense beam will be presented.
\end{abstract}

\section{Polarized proton beams in AGS and RHIC.}

The polarized beam for RHIC spin physics experimental program is produced in the Optically-Pumped Polarized $\mathrm{H}^{-}$Ion Source (OPPIS) [1]. The present RHIC OPPIS produces $0.5-1.0 \mathrm{~mA}$ (maximum $1.6 \mathrm{~mA}$ ) current in 400 $\mu$ s pulse duration. The polarized $\mathrm{H}^{-}$ion beam (of $35 \mathrm{keV}$ beam energy out of the source) is accelerated to 200 $\mathrm{MeV}$ in a 1 inear accelerator for strip-injection to Booster. $\mathrm{T}$ he $\mathrm{H}^{-}$ion pulse is captured in a single Booster bunch which contains about $4 \cdot 10^{11}$ polarized protons. The single bunch is accelerated in the Booster to $2.5 \mathrm{GeV}$ beam energy and then transferred to the AGS, where it is accelerated to $24.3 \mathrm{GeV}$ for injection to RHIC. RHIC is the first collider where the "Siberian snake" technique was very successfully implemented to suppress the resonance depolarization during beam acceleration in AGS and RHIC [2]. A luminosity of a $1.610^{32} \mathrm{~cm}^{-2} \mathrm{sec}^{-1}$ for polarized proton collisions in RHIC will be produced by colliding 120 bunches in each ring at $2 \cdot 10^{11}$ protons/bunch intensity.

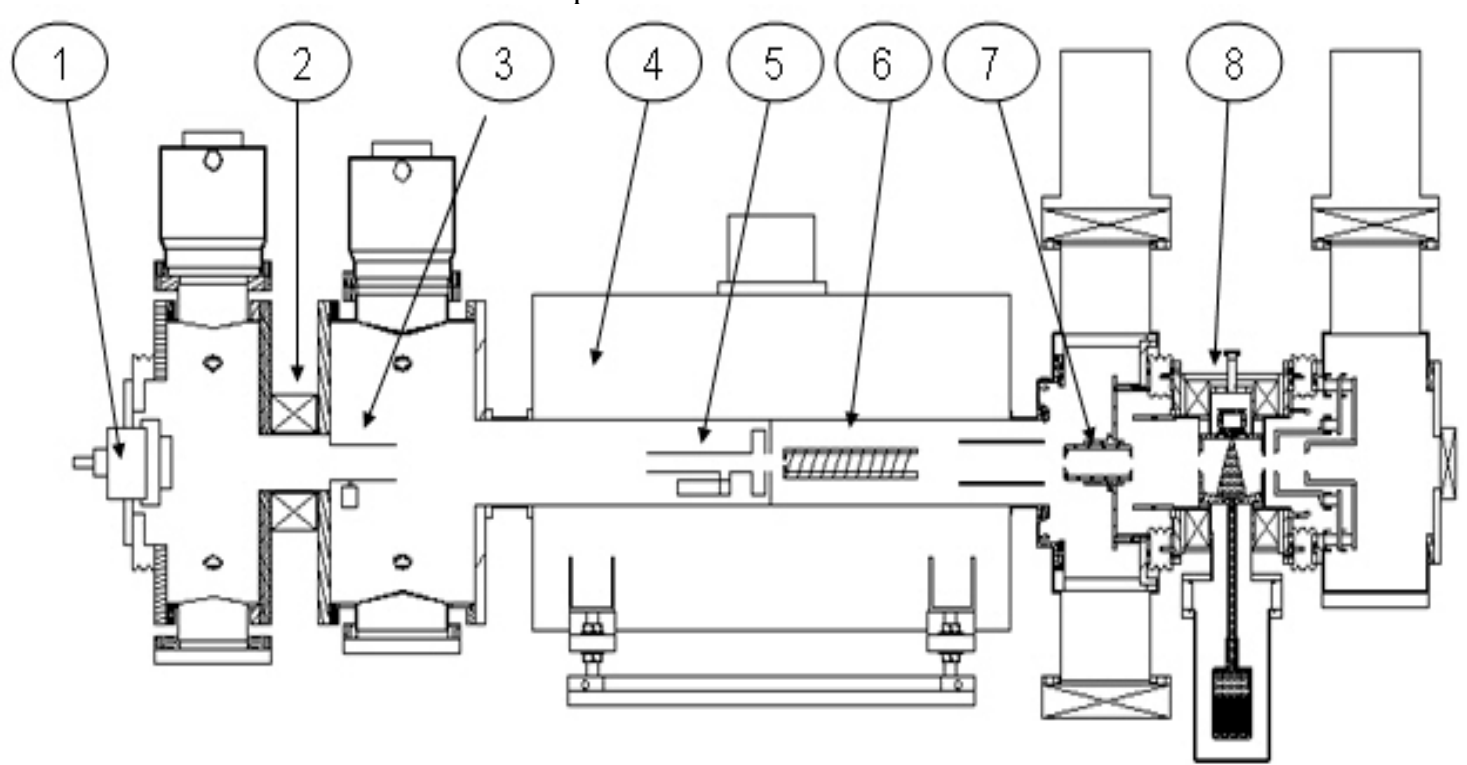

Fig. 1: Layout of the OPPIS with atomic hydrogen injector: 1-high-brightness proton source; 2- focusing solenoid; 3-pulsed hydrogen neutralization cell;4- super conducting solenoid $30 \mathrm{kG}$; 5-Pulsed He ionizer cell; 6optically-pumped Rb cell; 7-Sona shield; 8-sodium-jet ionizer cell. 
The RHIC polarized $\mathrm{H}^{-}$ion source is being upgraded to higher intensity (5-10 $\mathrm{mA}$ ) and polarization by using a very high brightness fast atomic beam source developed at BINP, Novosibirsk. This beam will be used in the RHIC polarization physics program at enhanced luminosity RHIC operation. The higher beam peak intensity will allow reduction of the transverse beam emittance at injection to AGS (by scraping of beam tails) to reduce polarization losses in AGS. There is also a plan the RHIC luminosity upgrade by using the electron beam lens to compensate the beam-beam interaction at collision points. This upgrade is also essential for future BNL plans for a high-luminosity electron - proton (ion) Collider eRHIC. In addition, the feasibility of high intensity (a few hundred $\mathrm{mA}$ ) high brightness unpolarized $\mathrm{H}^{-}$ion beam production in charge-exchange of high-brightness atomic hydrogen beam in sodium-jet ionizer cell will be studied.

A new polarimeter for absolute proton beam polarization measurements at $200 \mathrm{MeV}$ to accuracy better than $\pm 0.5 \%$ has been developed as a part of the RHIC polarized source upgrade. The polarimeter is based on the elastic proton-carbon scattering at a $16.2^{\circ}$ angle, where the analyzing power is large $99.35 \%$ and was measured with high accuracy [3].

\section{OPPIS upgrade with the Atomic Hydrogen Beam Injector.}

The ECR proton source is operated in high magnetic field. It has low hydrogen gas consumption, which makes possible a dc OPPIS operation with intensity in excess of $1.0 \mathrm{~mA}$. The proton beam produced in the ECR source has a co mparatively low emission current density and high beam divergence. This limits further current increase and gives rise to inefficient use of the available laser power for optical pumping. In fact only about $15 \%$ of the electron-spin polarized hydrogen atoms produced in $\mathrm{Rb}$ cell is within the ionizer cell acceptance.

In pulsed operation, suitable for application at highenergy accelerators and colliders, the ECR source limitations can be overcome by using a high brightness proton source outside the magnetic field instead of ECR[4,5]. Following neutralization in hydrogen, the high brightness $6.0-10.0 \mathrm{keV}$ atomic $\mathrm{H}^{0}$ beam is injected into a superconducting solenoid, where both a $\mathrm{He}$ ionizer cell and an optically-pumped $\mathrm{Rb}$ cell are situated in the 25-30 kG solenoid field, which is required to preserve the electron-spin polarization. The injected $\mathrm{H}$ atoms are ionized in the He cell with $80 \%$ efficiency to form a low emittance intense proton beam, which enters the polarized $\mathrm{Rb}$ vapor cell (see Fig.1). The protons pick up polarized electrons from the $\mathrm{Rb}$ atoms to become a beam of electron-spin polarized $\mathrm{H}$ atoms (similar to
ECR based OPPIS). A negative bias of about 3.0-7.0 kV applied to the He cell decelerates the proton beam produced in the cell to the $3.0 \mathrm{keV}$ beam energy optimal for the charge-exchange collisions in the $\mathrm{Rb}$ and sodium cells. This allows energy separation of the polarized hydrogen atoms produced after lower energy proton neutralization in $\mathrm{Rb}$ vapor and residual hydrogen atoms of the primary beam.

Residual higher energy atoms will be neutralized with lower efficiency in $\mathrm{Rb}$ cell (due to cross-section decrease at higher energy) and un-polarized component will be further suppressed by lower $\mathrm{H}^{-}$ion yield at 5.0$8.0 \mathrm{keV}$ atomic beam energy. The $\mathrm{H}^{-}$ion beam acceleration (by $-32 \mathrm{kV}$ pulsed voltage applied to the ionizer cell) will produce polarized $\mathrm{H}^{-}$ion beam of a 35 $\mathrm{keV}$ beam energy and un-polarized beam of a $40-43 \mathrm{keV}$ beam energy. Further suppression of un-polarized higher energy ion beam can be done in the LEBT.

Atomic hydrogen beam current of equivalent densities in excess of a $100 \mathrm{~mA} / \mathrm{cm}^{2}$ can be obtained at the $\mathrm{Na}$ jet ionizer location (about $200 \mathrm{c} \mathrm{m}$ from the source) by using a high brightness fast atomic beam source. This was tested in experiments at TRIUMF, where more than $10 \mathrm{~mA}$ polarized $\mathrm{H}^{-}$and $50 \mathrm{~mA}$ proton beam intensity was demonstrated [5]. $\mathrm{H}$ igher polarization is also expected with the fast atomic beam source due to: a) elimination of neutralization in residual hydrogen; b) better Sona-transition transition efficiency for the smaller $\sim 1.5 \mathrm{~cm}$ diameter beam; c) use of higher ionizer field (up to $3.0 \mathrm{kG}$ ). All these factors combined will further increase polarization in the pulsed OPPIS to $90 \%$ and the source intensity to over $10 \mathrm{~mA}$.

\section{Charge-exchange collisions. Beam energy optimization.}

The primary beam energy optimization is an important part of this development. Higher intensity and lower proton beam divergence can be obtained at higher beam energy. The neutralization efficiency in hydrogen cell is about $95 \%$ for energies $6-10 \mathrm{keV}$. There is a molecular $\mathrm{H}_{2}{ }^{+}$component (of about $5-10 \%$ ) in the extracted beam, which is dissociated in collisions with hydrogen in neutralizer cell producing atomic beam of one half (3-5 keV) beam energy. The ionization efficiency in He-ionizer cell is $80 \%$ at $6 \mathrm{keV}$ and $60 \%$ at $10 \mathrm{keV}$ beam energy. The proton beam produced in the $\mathrm{He}$ cell is decelerated to $3.0 \mathrm{keV}$ by the negative potential 3-7 keV applied to the cell.

At the $3.0 \mathrm{keV}$ beam energy the $\mathrm{H}^{-}$ion yield in the sodium ionizer cell is near maximum $(\sim 8.4 \%)$ and cross-section of polarized electron capture cross-section from $\mathrm{Rb}$ atoms is near maximum $\left(\sim 10^{-14} \mathrm{~cm}^{2}\right)$ too. The deceleration is produced by precisely aligned (to reduce 
beam losses) three-grid system. A small negative bias will be applied to the first grid and cylindrical electrode at the cell entrance to trap electrons in the cell for spacecharge compensation. The expected beam losses at the grids should not exceed $5-10 \%$. The beam divergence increase and associated losses will be studied experimentally.

About $40 \%$ residual (which passed the He-cell without ionization) atomic beam component of 6-10 keV energy will pass deceleration system, $\mathrm{Rb}$ cell (almost unaffected) and ionized in Na-cell producing $\mathrm{H}^{-}$ion beam. The $\mathrm{H}^{-}$ion yield at $6 \mathrm{keV}$ is about $5 \%$ and at 10 $\mathrm{keV}$ it is $\sim 2 \%$. $\mathrm{T}$ his is a significant suppression in comparison with main $3.0 \mathrm{keV}$ beam, but it would be a strong polarization dilution unless further suppression is applied.

The "velocity-filter" was developed for suppression of the lower $33.5 \mathrm{keV}$ beam energy component produced at dissociation of molecular ions in the ECR primary proton source. The "filter" is a double Einzel lens system, which was installed in the OPPIS LEBT. A negative potential of about $35 \mathrm{keV}$ is applied to the first lens. This potential decelerates and retards the lower energy (un-polarized) beam component. The second Einzel lens is tuned to compensate the strong focusing of the first lens for optimal beam transmission further for injection to RFQ. This velocity "filter" suppresses not only low energy but also higher energy beam components. The suppression factor exceeds order of magnitude for the beam energy difference $2 \mathrm{keV}$. For energy difference $7 \mathrm{keV}$ the estimated suppression will be about 100 times and polarization dilution should be less than $0.3 \%$.

At $6.0 \mathrm{keV}$ primary beam energy the dissociation of molecular $\mathrm{H}_{2}{ }^{+}$ions will produce $3.0 \mathrm{keV}$ atomic hydrogen beam. About $20 \%$ of this beam will pass the He-cell without ionization and will be converted to $\mathrm{H}^{-}$ ion beam of the same energy as main polarized beam (no further suppression can be applied in the LEBT). In assumption that $\mathrm{H}_{2}{ }^{+}$from the source is about $5 \%$ the polarization dilution would be $2.5 \%$. The use of higher energy $10 \mathrm{keV}$ beam would be beneficial since higher energy $5 \mathrm{keV}$ beam is produced after dissociation and dilution component energy is $37 \mathrm{keV}$. This $2 \mathrm{keV}$ difference is sufficient for ten-fold suppression by double EL system in LEBT and polarization dilution can be reduced to $0.25 \%$.

\section{Atomic beam source development.}

The atomic beam injector is under development at BINP, Novosibirsk. In this injector the proton beam is produced by a four-grid multi-aperture ion extraction optical system and neutralized in the $\mathrm{H}_{2}$ gas cell downstream from the grids. A highbrightness atomic hydrogen beam was obtained in this injector by using a plasma emitter with a low transverse ion temperature of $\sim 0.2 \mathrm{eV}$ which is formed by plasma jet expansion from the arc plasma generator [7]. The multi-hole grids are spherically shaped to produce "geometrical" beam focusing [8]. The grids are made of $0.2 \mathrm{~mm}$ thick molybdenum plates. Holes in the plates (of a 0.4 $\mathrm{mm}$ in diameter) were produced by photo-etching techniques. An array of 7466 holes is forming a hexagonal structure with the step of $0.55 \mathrm{~mm}$ and outer diameter of $5.0 \mathrm{~cm}$. The grids were shaped by re-crystallization under pressure at high temperature. The grids are welded to stainless steel holders by pulsed $\mathrm{CO}_{2}$ laser. At emission current density of a $470 \mathrm{~mA} / \mathrm{cm}^{2}$ an angular divergence of the produced beam is $\sim 15 \mathrm{mrad}$.

The focal length of the spherical ion extraction system was optimized for OPPIS application, which is characterized by a long polarizing structure of the charge-exchange cells and small $(2.0 \mathrm{~cm}$ in diameter) Na-jet ionizer cell, which is located at a $240 \mathrm{~cm}$ distance from the source (see Fig. 1).

An optimal drift-space length of about $130 \mathrm{~cm}$ is required for convergence of the $5 \mathrm{c} \mathrm{m}$ (initial diameter) beam to $2.5 \mathrm{~cm}$ diameter He-ionizer cell. After ionization in the He-cell the proton beam gyro-center is not moving radially, while the ions travel in uniform magnetic field inside the solenoid, and proton velocities remain the same. Therefore, the protons do not experience any angular divergence for about $70 \mathrm{~cm}$ from the end of the He ionizer cell to the end of the solenoid and the magnetic field conserves the current density profile and the beam angular divergence. After the solenoid the divergence becomes larger due to addition of the randomized regular radial motion to the initial inherent divergence of the emitter, but the $40 \mathrm{~cm}$ distance to the Na-jet cell is relatively short, and the resulting ion beam expansion remains acceptable. Thus, in the given geometry the effect of the "current density conservation" in the magnetic field occurs to be stronger than the increased angular divergence after the solenoid. With the magnetic field the total 
current through the Na-jet cell is by a factor of 2.3 larger than the current in the absence of magnetic field in the same geometry.

About 10\% (of total neutral injector current of a 4 A) can be transported through the Na-jet cell acceptance (with the magnetic field) by using optimal extraction grid system of a focal length: $F \approx 200 \mathrm{~cm}$. Taking into account ionization efficiency in He-cell of a $60 \%$, polarized electron capture in the $\mathrm{Rb}$-cell of a $50 \%$ and $\mathrm{H}^{-}$yield in the Na-jet cell of a $8.4 \%$ the expected polarized $\mathrm{H}^{-}$ion beam current is expected to be $\sim 10 \mathrm{~mA}$. The beam deceleration after the He-cell may introduce some additional beam losses, which will be studied experimentally.

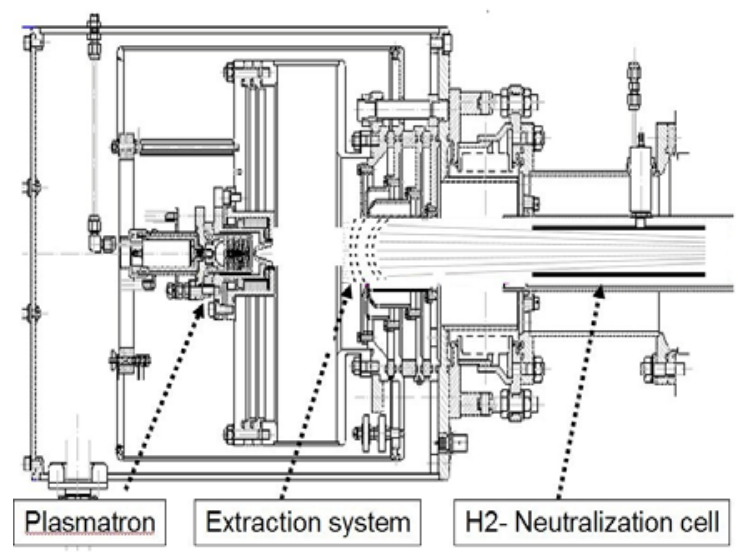

Figure 2. Atomic beam injector with geometrical focusing.

\begin{tabular}{|c|c|c|c|}
\hline IOS \# & $\begin{array}{c}\text { Beam equivalent } \\
\text { current density } \\
(\mathrm{mA} / \mathrm{cm} 2)\end{array}$ & $\begin{array}{c}\text { Angular } \\
\text { divergence } \\
(\mathrm{mrad})\end{array}$ & $\begin{array}{c}\text { Focal } \\
\text { length } \\
(\mathrm{cm})\end{array}$ \\
\hline 1 & 340 & 12 & 166 \\
\hline 2 & 320 & 7 & 300 \\
\hline 3 & 300 & 8 & 270 \\
\hline
\end{tabular}

\section{Experimental results.}

Studies of the neutral beam formation and chargeexchange processes are presently in progress at the fullscale Test Bench, which is closely reproduce the OPPIS upgrade Layout (see Fig.1) except the superconducting solenoid, which is replaced by a cy lindrical vacuum chamber of $150 \mathrm{~mm}$ ID. A four electrode multi-wire ion optical system developed for experiments at TRIUMF [5] was initially used for these studies. The proton beam of 3-7 keV energy and total current of 3-6 A is focussed by solenoid lens and then is neutralized in the pulsed hydrogen target. Atomic hydrogen beam is ionized in the Na-jet cell and is deflected by bending magnet and measured by the Faraday cup in the diagnostic box. The maximum $\mathrm{H}^{-}$beam current of a $12 \mathrm{~mA}$ at $7 \mathrm{keV}$ beam energy was obtained in these experiments (see Fig. 3). Taking into account that $\mathrm{H}^{-}$ion yield is $\sim 4 \%$ at energy $7 \mathrm{keV}$ the total equivalent neutral hydrogen beam intensity was estimated at $300 \mathrm{~mA}$. The estimated angular divergence of the formed atomic beam (from beam profile measurements by the secondary emission monitor) was $\sim 15 \mathrm{mrad}$ normalized emittance of the produced $\mathrm{H}^{-}$beam is estimated as $0.15 \cdot \mathrm{cm} n \mathrm{ad}$, as

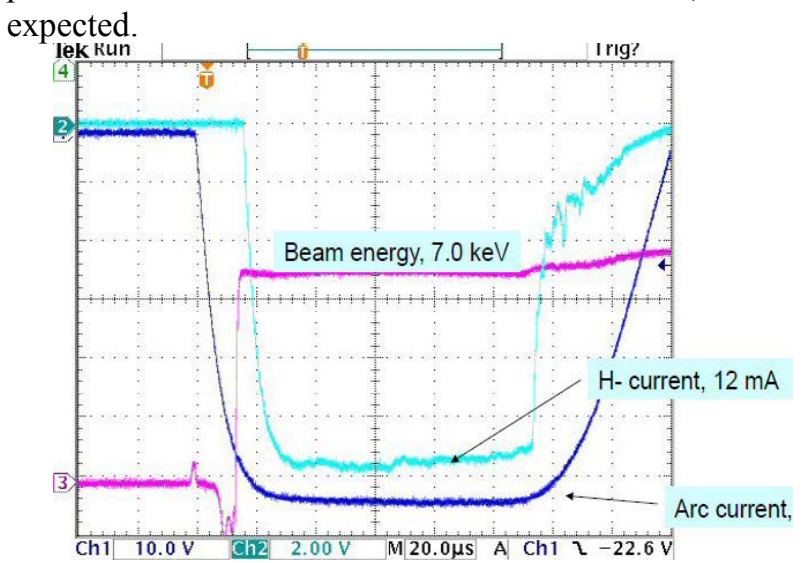

Figure 3. Oscillogram of the $\mathrm{H}^{-}$ion current, arc current (600 A), and extraction volta.ge pulse $(7 \mathrm{keV})$.

\section{Summary.}

Polarized $\mathrm{H}^{-}$ion beam current in excess of $10 \mathrm{~mA}$ is expected after the OPPIS upgrade with this Atomic Hydrogen Injector developed at BINP, Novosibirsk. Higher polarization is also expected with the fast atomic beam source due to: a) elimination of neutralization in residual hydrogen; b) better Sona-transition transition efficiency for the smaller $\sim 1.5 \mathrm{~cm}$ diameter beam; c) use of higher ionizer field (up to $3.0 \mathrm{kG}$ ). The beam emittance will be kept below $2.0 \pi \mathrm{mmmrad}$ due to the smaller beam diameter. All these factors combined will increase polarization in the pulsed OPPIS to $\sim 85-90 \%$.

The RHIC polarized source upgrade for higher intensity and polarization is funded for implementation in 2009-12. The source will provide the high intensity low emittance beam for polarized RHIC luminosity upgrade and for future eRHIC facilities.

\section{References}

1. Zelenski, A., et al., Rev. Sci. Instr.. 그, pp. 888891 (2002)

2. T. Roser, AIP Conf. Proc, 980, p.15, (2008).

3. A.Zelenski et al., this conference. 
4. A.Zelenski et al., NIM A242, p.223,(1986)

5. A. Zelenski et al., AIP Conf. Proc. 570, p.179, (2000).

6. A.Zelenski et al., SPIN 2002 Proc, AIP Conf. Proc., 675, p.881, (2003)

7. V.I.Davydenko et al., NIM A427, p.234 (1999) .

8. V.I Davydenko, A.A.Ivanov, Rev. Sci.Instr., 72, p.1809,(2004). 